\title{
SISTEMA DE CONTROLE PARA ESCADA ROLANTE
}

\section{CONTROL SYSTEM FOR ESCALATOR}

\section{Fábio Luís Narduci ${ }^{1}$; Luciano Akiyoshi Aoki ${ }^{2}$ Mauricio Ginez Romero $^{3}$; Claiton Moro Franchi ${ }^{4}$}

${ }^{1}$ Graduado em Tecnologia de Automação Industrial, Centro Universitário de Maringá

- CESUMAR, Maringá-PR, e-mail: fnarduci@hotmail.com

${ }^{2}$ Graduado em Tecnologia de Automação Industrial, Centro Universitário de Maringá

- CESUMAR, Maringá-PR, e-mail: luciano_aoki@msn.com

${ }^{3}$ Graduado em Tecnologia de Automação Industrial, Centro Universitário de Maringá

- CESUMAR, Maringá-PR, e-mail: polotex@uol.com.br

${ }^{4}$ Professor Mestre, Depto de Engenharia de Controle e Automação e Automação

Industrial, Centro Universitário de Maringá - CESUMAR, Av. Guedner 1610, Jardim

Aclimação, CEP 87050-390 Maringá-PR, e-mail: claiton@cesumar.br

\section{RESUMO}

Muitos estabelecimentos comerciais utilizam escadas rolantes que desempenham sua função em regime normal de funcionamento. Existe um grande desperdício de energia elétrica, já que em boa parte não há transporte de usuário. A busca por novas fontes não deve ser a única preocupação no contexto dos recursos energéticos, mas também o desenvolvimento de novas tecnologias que visam o uso otimizado destes recursos. Nesta conjuntura, este projeto visa implantar um sistema de controle para escadas rolantes, a fim de otimizar seu uso e reduzir o consumo de energia do equipamento. $\mathrm{O}$ funcionamento do sistema baseia-se no princípio de que, decorrido um tempo $t_{A}$ sem fluxo de usuário, tempo este previamente definido por uma análise estatística, a escada reduz sua velocidade; se transcorrido um tempo $t_{B}$, sendo $t_{B}>t_{A}$, a escada é parada. Havendo presença de usuário, a escada apresenta aceleração suave, restabelecendo o regime normal de funcionamento sem riscos de utilização. A economia de energia surge quando a escada está parada ou trabalhando com velocidade reduzida. Este projeto consiste em substituir o comando elétrico do tipo reversor e com partida direta (FRANCHI, 2007), pelo sistema de controle proposto. A redução do consumo de energia foi estimada realizando-se uma análise estatística. Por meio deste pode-se maximizar o aproveitamento do sistema e estipular as faixas de economia para escadas de alto e baixo fluxo de usuários. Este sistema é ecologicamente correto e contribui para a otimização do consumo de energia elétrica. Palavras-chave: Economia de energia. Retorno Rápido. Controle de velocidade. Ecologicamente correto. Redução do consume. 


\section{ABSTRACT}

Many business stablisment utilize escalator that performs it's function in a regular way of work. There is a big waste of eletric power, as in a good part of it there isn't a transportation of user. The search for new sources shouldnt't is the only worry in the context of energetic resource, but also the development of new technologies aimed an optimal use of this recourse. In the conjuncture, this project aims to establish a escalators' control system, to optimize their use and reduce energy consumption of equipment. The system's operation is based on the principle that, after a time $t_{a,}$, a free flow of user, which this time is previously define by a statistician study the escalator reduce its speed, if it has passed a time $t_{b}$, being $t_{b}>t_{a}$, the escalator is stopped. If we have the presence of the user, the escalator presents low acceleration, establishing the regular way of work without risks of utilization. The energetic economy happens when the escalator is stopped, or working with reduced speed. The project consists in replace the eletric command of the reversor and with the direct start, by a system's control proposed. The reduction of the energy comsumption was valued by a statistician study, so trough it we can increase the use of the system and stipulate of the economy zone for the escalators with high and low user's flow. This system is ecologically correct and contribute for the consumption optimized of electric power.

Keywords: Energy saving. Quick return. Speed control. Ecologically correct. Reduction of expenses.

\section{INTRODUÇÃO}

O mundo está passando por efeitos catastróficos no meio ambiente, tais como derretimento de geleiras, aquecimento global, aumento na incidência de furacões, enchentes, entre outras. Esta realidade tem gerado grandes discussões em torno da necessidade de economia de energia como forma de otimização de desempenho das organizações que a utilizam e que, com a redução do consumo, acabam por diminuir os impactos ambientais decorrentes da sua geração Agência Nacional de Energia Elétrica (ANEEL, 2007).

A busca por novas fontes alternativas de energia, tais como o Biodiesel e o hidrogênio combustível, não são as únicas medidas a serem tomadas para alcançar os objetivos. A implantação de tecnologias em máquinas e equipamentos, afim do melhor aproveitamento de energia, torna-se também necessária.

As escadas rolantes estão espalhadas em diversos ramos da economia. Muitos comércios, aeroportos, shoppings e metrôs, utilizam escadas rolantes para facilitar a circulação das pessoas em seu recinto. As escadas possuem um considerável consumo, visto que estas trabalham em regime normal, isto é, com velocidade máxima e de forma constante, durante todo expediente. Este fato provoca um grande 
desperdício de energia elétrica, já que em boa parte do seu funcionamento não estão transportando usuários, elevando os gastos das contas de energia e condomínio dos estabelecimentos.

O intuito deste trabalho é apresentar o Sistema de Controle para Escadas Rolantes (SCER), cujo objetivo é minimizar o consumo de energia elétrica por meio da diminuição dos períodos de regime normal de funcionamento. Para isso, busca-se criar um sistema que reduza a velocidade da escada quando não estiver transportando pessoas em períodos de maior circulação de usuários, e que deixe a escada sem funcionamento, em "modo de espera", em horários de movimento reduzido, quando não solicitada. Neste contexto a implantação deste sistema melhorará a relação custo benefício, já que somente funcionará em regime normal quando necessitada.

A análise estatística realizada definirá os horários de maior circulação e o número de pessoas que transitam por hora em um shopping da cidade de Maringá, PR. Este estudo deverá mostrar o tempo mínimo para que a escada desligue ou reduza a velocidade, caso esteja rodando sem circulação de pessoas. Esta análise também é importante para que se possa estabelecer um tempo de segurança para que o sistema não fique ligando e desligando, o que provocaria desgaste no equipamento e facilitar os cálculos para uma estimativa de economia de energia.

\section{METODOLOGIA}

O SCER é composto por um sensor de presença, um circuito eletrônico microcontrolado (BOYLESTAD; GUIMARÃES e OLIVEIRA, 1999) e um inversor de frequência, como apresentado na Figura 1. Ao detectar a presença de usuários, o sensor de presença envia um sinal digital ao circuito microcontrolado que estabelece o regime normal de funcionamento propiciado pelo inversor de frequência. A linguagem Assembly (ZANCO, 2005) foi utilizada para a programação do Microcontrolador PIC16F628, que faz o processamento dos dados.

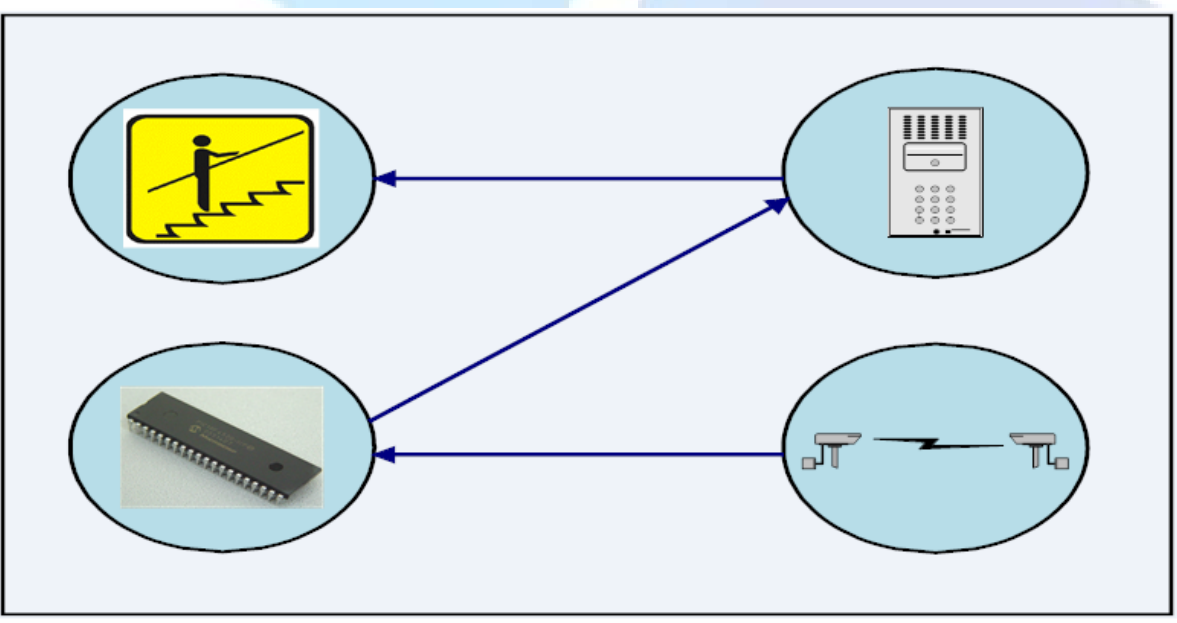

Figura 1. Integração dos Componentes utilizados no controle SCER 
Ao iniciar o funcionamento da escada, o sistema entra em regime normal de funcionamento. Se decorrido um tempo $\mathrm{A}\left(\mathrm{t}_{\mathrm{a}}\right)$ sem fluxo de usuários, isto é, sem o sensor de presença ser acionado (Nível lógico 1), a velocidade da escada é reduzida por meio da diminuição da frequência do inversor, modificação esta que será solicitada pelo circuito eletrônico microcontrolado. A cada entrada digital do inversor está associado um estado de funcionamento da escada, sendo possível três estados: regime normal de funcionamento, regime reduzido de funcionamento e regime parado (esteira com velocidade nula). Se transcorrido um tempo $B\left(t_{b}\right)$, sendo $t_{b}>t_{a}$, estando a escada com sua velocidade reduzida e sem passagem de usuários, a escada entra em regime parado. Havendo presença de usuário, a escada apresenta aceleração suave, restabelecendo o regime normal de funcionamento sem riscos de utilização. A Figura 2 apresenta um fluxograma de funcionamento do sistema.

Sabe-se que quando um motor de indução trabalha por longos períodos em baixas frequências pode ocorrer uma elevação de temperatura, o que poderia causar danos aos seus componentes. A fim de evitar estas avarias, o circuito eletrônico possui uma entrada digital para receber os sinais de um sensor de temperatura. A correção da temperatura é feita com o estabelecimento do regime normal de funcionamento, aumentando assim a ventilação do motor.

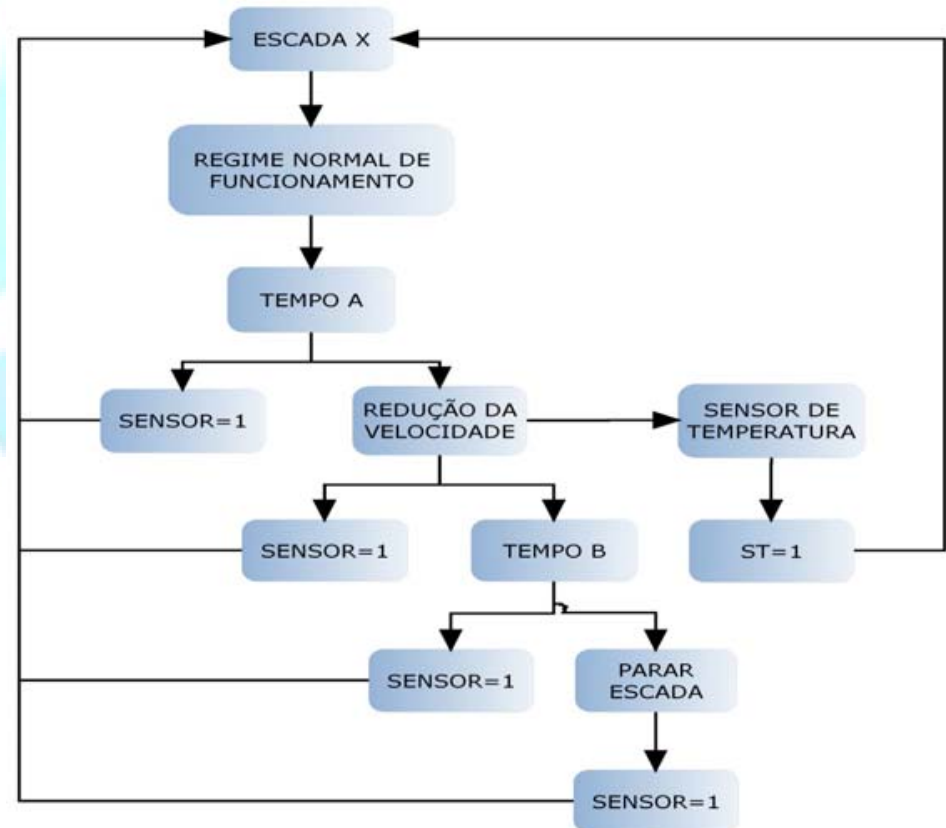

Figura 2. Fluxograma de funcionamento do SCER

O comando elétrico da escada rolante é definido como reversor e sua partida é do tipo direta. É importante lembrar que a partida direta proporciona elevados picos de corrente. As escadas rolantes fazem uso de dois painéis de acionamento, sendo eles o Painel Principal de Acionamento, responsável pela partida e reversão do sentido de fluxo da escada, e o painel de segurança, que controla os dispositivos 
mecânicos através de sensores fim-de-curso. O Painel Principal de Acionamento será substituído pelo SCER, utilizando apenas o painel de segurança da escada.

Uma análise estatística foi desenvolvida para facilitar a determinação dos tempos $t_{a}$ e $t_{b}$, auxiliar na estimativa das faixas de economia e definir o fluxo de usuários. Este estudo foi baseado na Teoria das Filas (PRADO, 2006), onde os sistemas de filas são caracterizados por cinco componentes: modelo de chegada dos usuários, modelo de serviço, número de atendentes, capacidade do estabelecimento para atender usuários e ordem em que os usuários são atendidos.

O modelo de chegada dos usuários é usualmente especificado pelo tempo entre chegadas; já o número de atendentes para o estudo realizado é um, visto que a análise é realizada para uma escada. A capacidade de atendimento de usuários da escada por unidade de tempo é definida pelo fabricante.

A escada estudada é representada por um sistema de fluxo aleatório, ou seja, não é possível predizer como vai se comportar a demanda de serviço, justificando-se a utilização desta teoria. $O$ processo de chegada de usuários é apresentado pelos instantes $t_{1}, t_{2}, t_{3}, \ldots, t_{n}$, sendo os intervalos entre chegadas $(\tau)$ igual $a$ :

$$
\tau_{\mathrm{n}}=\mathrm{t}_{\mathrm{n}}-\mathrm{t}_{\mathrm{n}-1}
$$

Outra característica do sistema abordado é que a disciplina das filas analisada é do tipo FIFO (First In, First Out), uma vez que o primeiro usuário a entrar na fila deve ser o primeiro a sair da escada.

O modelo de serviço é normalmente especificado pelo tempo de serviço. Este período é o tempo requerido pela escada para atender um usuário, isto é, tempo que o usuário está sendo transportado pela escada, sendo para a escada estudada de oito segundos.

A coleta dos dados para a análise estatística foi feita em um shopping durante todo o expediente de uma sexta-feira (horário compreendido entre 10:00 horas e 01:00 do dia seguinte). A escada rolante analisada foi à indicada pela diretoria do estabelecimento como sendo a de maior circulação. Assim, a economia apresentada para esta escada estaria considerando o pior caso, visto que a escada apresentada possui um alto fluxo de usúario, o que minimizaria os tempos nos quais o sistema estaria em regime reduzido ou em regime parado.

A economia obtida com a implantação do SCER surge quando a escada está trabalhando em regime reduzido de velocidade ou em regime parado, tendo assim uma redução da frequência no motor de indução, que é a maior fonte consumidora de energia do conjunto. Este fato é propiciado pelo uso do inversor de frequência. $\mathrm{O}$ consumo de energia em motores de indução é diretamente proporcional aos valores de corrente elétrica e tensão (SIMÃO e ALMEIDA NETO, 2002). Quando o motor trabalha em frequências reduzidas a sua corrente elétrica e tensão são também diminuídas, reduzindo assim a demanda de energia.

Nas Figuras 3 e 4 são apresentados para exemplificar a variação dos valores de tensão e corrente em relação à frequência em motores de indução, onde os valores de 
frequência foram alterados por um inversor de frequência. Devido à dificuldade envolvida na aquisição dos valores das variáveis necessárias para realização do estudo, foram realizados experimentos para coletar as faixas de percentuais de economia de um motor de indução de $4 \mathrm{CV}$, levando em consideração a mesma proporção de carga apresentada no motor da escada analisada, que possui uma potência aproximada de $15 \mathrm{CV}$.

\section{RESULTADOS E DISCUSSÃO}

O SCER utiliza para o regime reduzido um frequência de $30 \mathrm{~Hz}$, e para o regime parado uma frequência de $0 \mathrm{~Hz}$. Assim, analisando-se as Figuras 3 e 4 nota-se que a economia em relação à corrente elétrica quando a frequência de trabalho está em $30 \mathrm{~Hz}$ é de aproximadamente $15 \%$, e a diminuição em relação à tensão elétrica aproxima-se de $48 \%$. Já para o regime parado subtende-se que como a frequência é de $0 \mathrm{~Hz}$, os valores de tensão e corrente são praticamente nulos. Estes resultados serão posteriormente adotados para o cálculo de estimativa de economia.

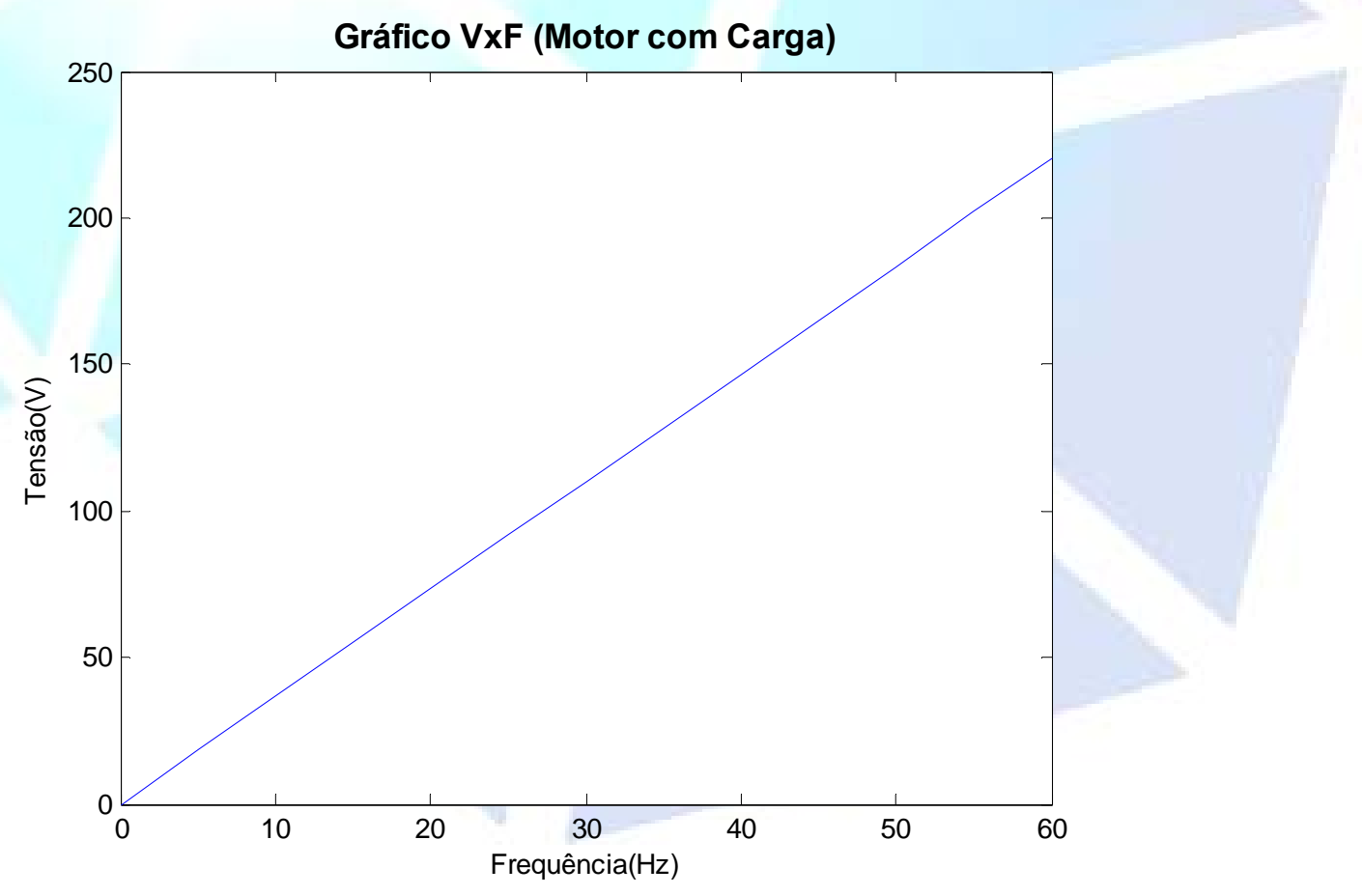

Figura 3. Gráfico FxV para motor de Indução de 4 cv. 


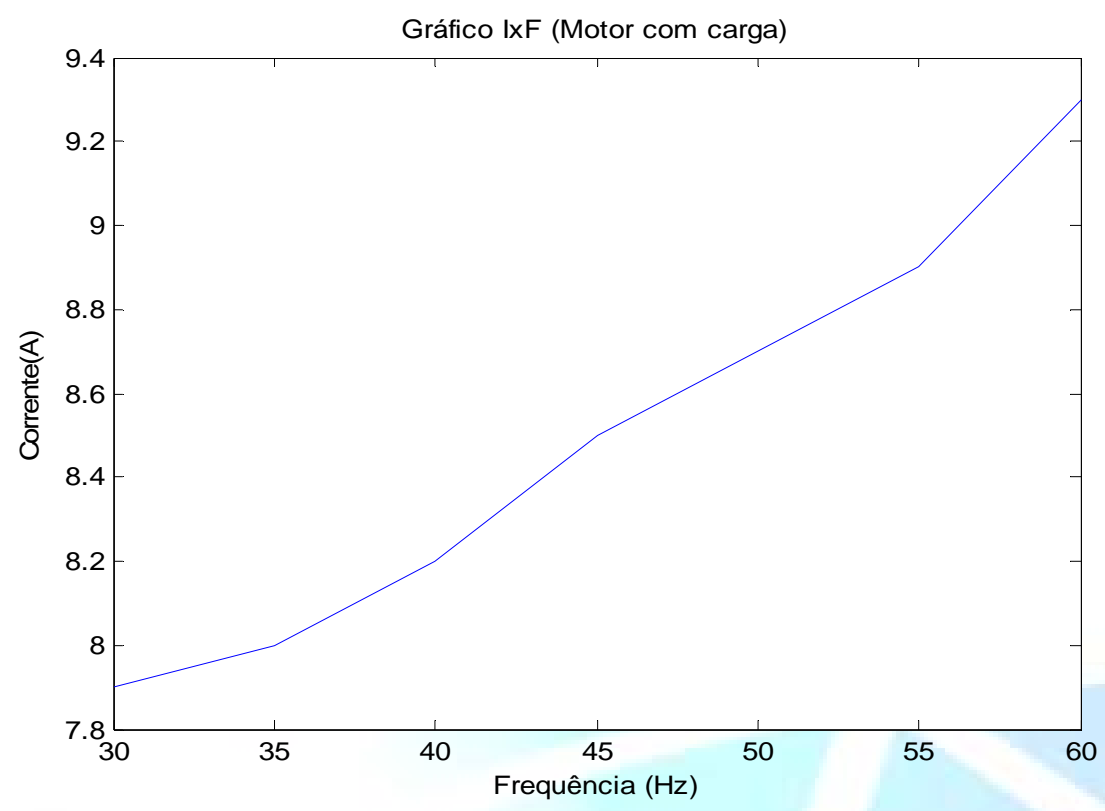

Figura 4. Gráfico FxI para motor de Indução de 4 cv.

Para que fosse possível a estimativa da economia obtida com a implantação do SCER foi necessário definir os tempos médios entre as chegadas de usuários (TMEC). Este período foi obtido por meio da Média das médias de cada intervalo de hora (como observado na Figura 5). O valor encontrado foi de 40 segundos para a escada estudada, assim ficando definido que em média o tempo entre a chegada de um usuário ou um grupo de usuários simultaneamente e outro é de 40 segundos. Este valor relativamente alto é justificado pelo baixo fluxo em períodos iniciais e finais de expediente (Figura 6), onde o SCER terá maiores faixas de aproveitamento.

Como o tempo médio entre as chegadas possui um valor considerado elevado, para otimizar o uso do sistema foi definido que o tempo $t_{a}$ para a escada estudada é de 10 segundos, visto que a escada demora 8 segundos para transportar um usuário em todo seu trajeto e para reduzir a velocidade a escada não necessita esperar longos períodos. Já o tempo $t_{b}$ foi definido como 60 segundos, período este maior que o TMEC. 


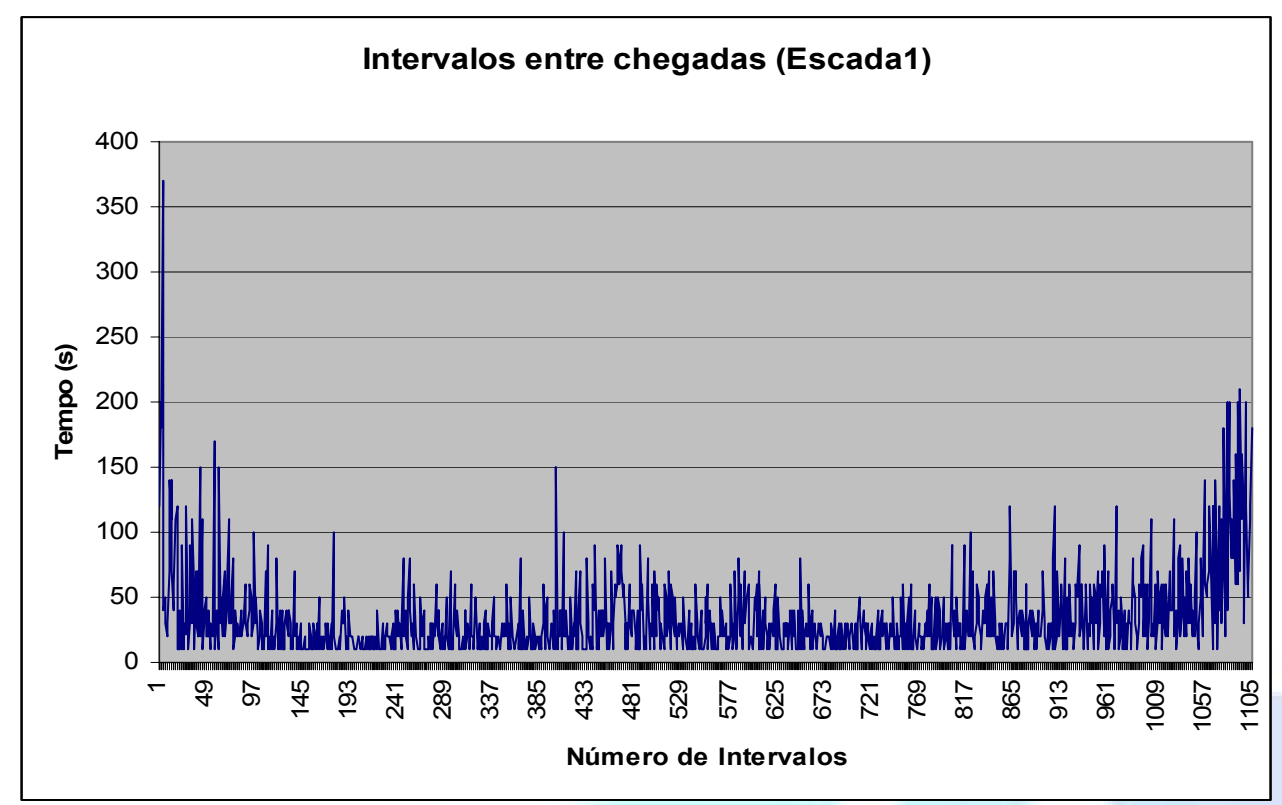

Figura 5. Intervalo entre chegadas

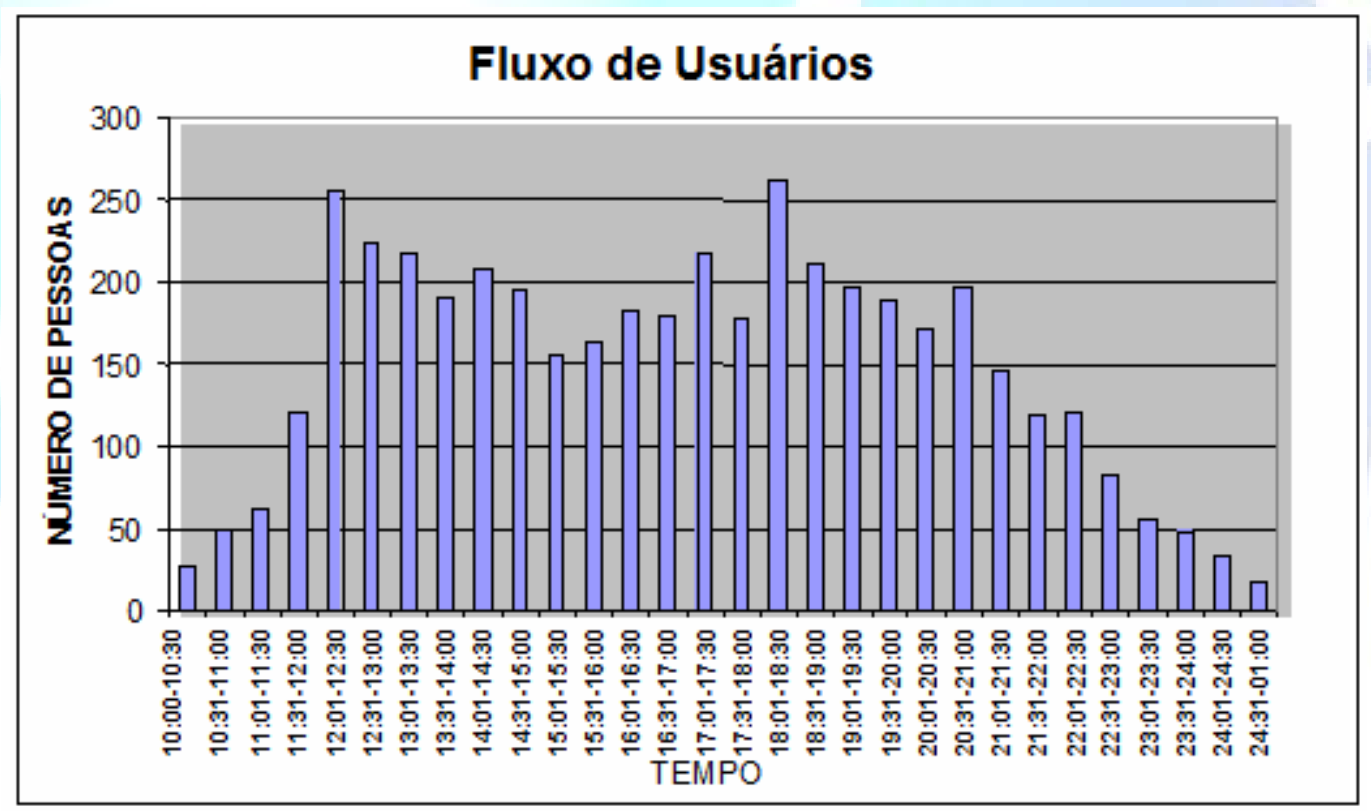

Figura 6. Fluxo de Usuários da escada analisada

Para estimar a economia de energia a análise foi realizada em dois casos, aquele em que o SCER trabalha em regime de funcionamento reduzido (RR) ou em regime parado (RP).

Para os cálculos do sistema em regime reduzido foram utilizadas as seguintes condições:

- Se o intervalo A for maior que 60 segundos, sem passagem de usuários, o valor acumulado será de 50 segundos, uma vez que o tempo seguinte aos 10 segundos 
o SCER estará em regime reduzido, e no tempo seguinte aos 60 segundos ele já entra em outra faixa de classificação, pois a escada estaria em regime parado;

- Caso o intervalo A seja menor que 60 segundos, o valor acumulado será o valor do intervalo subtraído de 10 segundos, pois a escada demora 10 segundos para entrar em RP. Pode-se representar ainda a equação em notação utilizada por planilhas do Microsoft EXCEL como: (SE(A>60;50;A-10)).

Realizando o somatório do tempo total no qual o sistema trabalhou RR (Figura 7) foi encontrado um valor de 5,92 horas. Assim neste período a escada estaria em regime reduzido de velocidade.

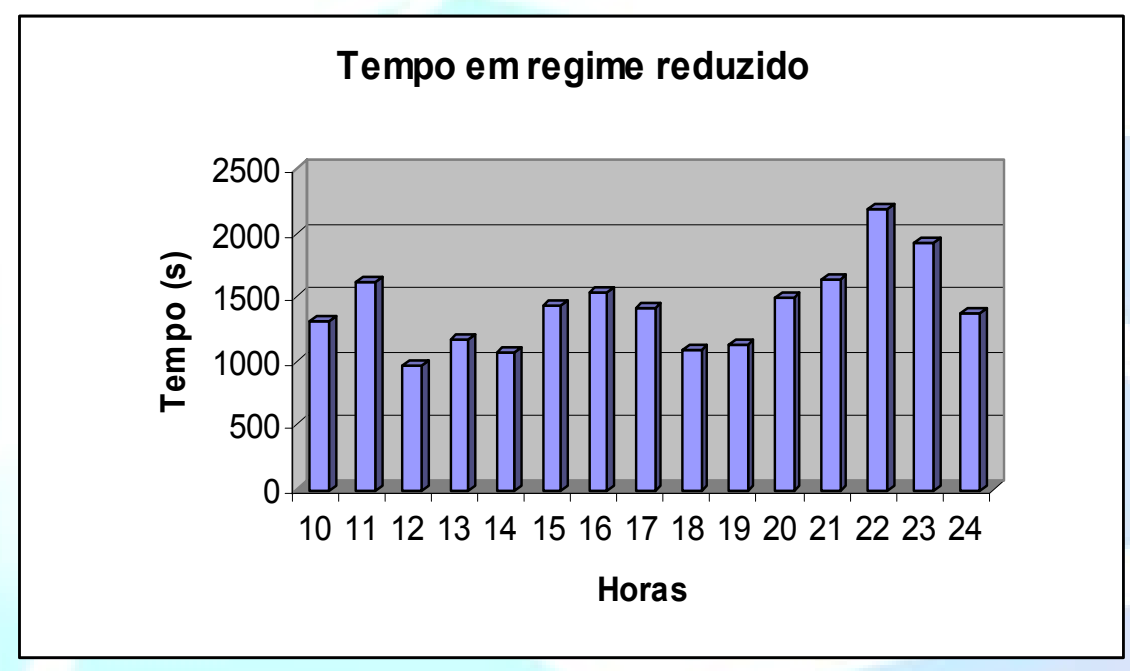

Figura 7. Intervalo em regime reduzido

A obtenção do período em que o sistema esteve em regime parado teve em consideração as seguintes hipóteses:

- Se o intervalo A for maior que 60 segundos, sem passagem de usuários, o tempo acumulado será o valor subtraído de 60, já que o período anterior aos 60 segundos o SCER está computado na faixa de classificação de RR;

- Caso o intervalo A seja menor que 60 segundos o valor acumulado será zero, pelo mesmo motivo anterior. Pode-se representar ainda a equação em notação utilizada por planilhas do Microsoft EXCEL como: (SE(A>60;A-60;0)).

O tempo total em que o sistema trabalhará em regime parado foi encontrado através do somatório dos intervalos ao longo do expediente (Figura 8). Para a escada analisada este tempo foi de 1,55 horas. Analisando a Figura 8 pode-se concluir que o tempo definido para que o sistema entre em RP é satisfatório, já que as maiores incidências desta situação ocorrem nos períodos de menor circulação, como mostra a Figura 6, o que implica em menor desgaste do equipamento com as partidas do motor. 


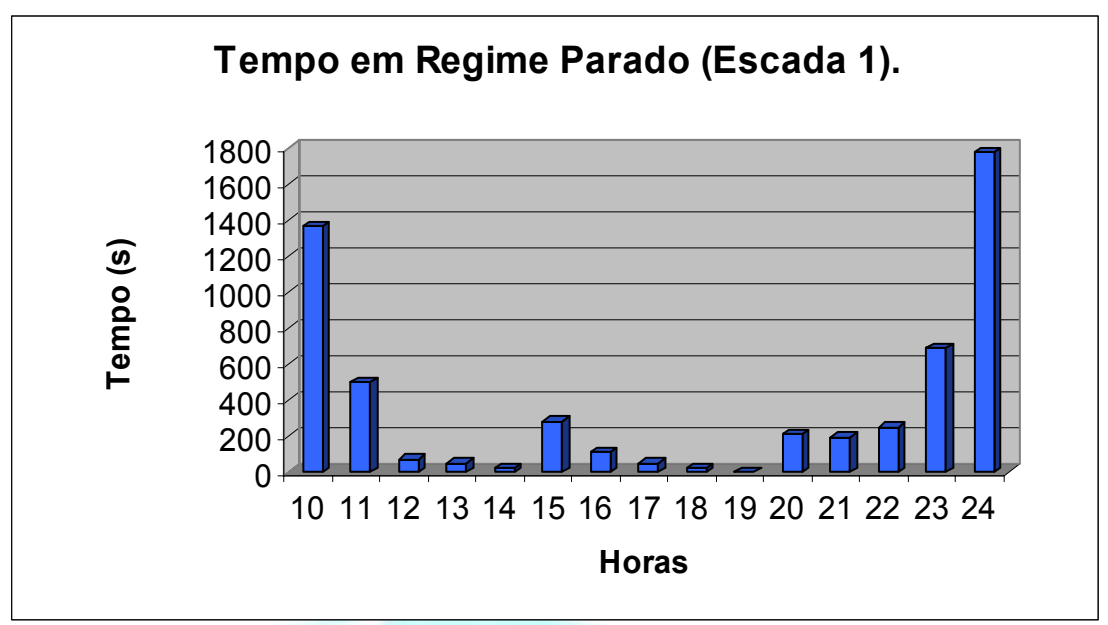

Figura 8. Intervalo em regime parado

A estimativa de economia a ser apresentada foi baseada na escada estudada. Para os cálculos foram coletados os dados do motor:

- Motor de 15Hp;

- Tensão 380V;

- Corrente 29 A;

- Fator de Potência 0,86.

$\mathrm{O}$ cálculo do consumo de energia em regime normal de funcionamento (CS), isto é, sem a implantação do SCER levando em consideração que a escada trabalha 14 horas por dia e o preço do KWh foi considerado R\$ 0,38 , têm-se:

$\mathrm{C}_{\mathrm{S}}=$ Consumo normal em Reais;

$\mathrm{P}=\mathrm{U} \times \mathrm{I} \times \mathrm{FP}$

$\mathrm{P}=380 \times 29 \times 0,86$

$\mathrm{P}=9,47 \mathrm{KW}$

$\mathrm{C}=\mathrm{P} \times \Delta \mathrm{t}$

$\mathrm{C}=9,47 \times 14 \times 30$

$\mathrm{E}=3977 \mathrm{KWh}$

$\mathrm{C}_{\mathrm{S}}=3977 \times 0,38=\mathrm{R} \$ 1511$

Para a definição do consumo da escada com a implantação do SCER devem-se obter os consumos parciais $\mathrm{C}_{\mathrm{N}}, \mathrm{C}_{\mathrm{R}}$ e $\mathrm{C}_{\mathrm{P}}$, descritos posteriormente. $\mathrm{O}$ consumo total $\left(\mathrm{C}_{\mathrm{T}}\right)$ é obtido pelo somatório dos parciais.

Os tempos nos quais a escada esteve em regime reduzido e com velocidade nula foram definidos anteriormente. Assim, presumisse que o tempo no qual a escada trabalha em regime normal será:

$\mathrm{T}_{\mathrm{N}=}$ Tempo em regime normal;

$\mathrm{T}_{\mathrm{R}=}$ Tempo em regime reduzido;

$\mathrm{T}_{\mathrm{P}=}$ Tempo parada;

$\mathrm{T}_{\mathrm{T}}=$ Tempo total de funcionamento;

$\mathrm{T}_{\mathrm{N}=} \mathrm{T}_{\mathrm{T}-} \mathrm{T}_{\mathrm{R}-} \mathrm{T}_{\mathrm{P}}$ 
$\mathrm{T}_{\mathrm{N}=} 14-5,92-1,55$

$\mathrm{T}_{\mathrm{N}=6,53 \text { horas. }}$

Para a escada em regime normal tem-se o seguinte consumo:

$\mathrm{C}_{\mathrm{N}}=$ Consumo em regime normal;

$\mathrm{C}_{\mathrm{R}}=$ Consumo em regime reduzido;

$\mathrm{C}_{\mathrm{P}}=$ Consumo em regime parado;

$\mathrm{P}=\mathrm{U} \times \mathrm{I} \times \mathrm{FP}$

$\mathrm{P}=380 \times 29 \times 0,86$

$\mathrm{P}=9,47 \mathrm{KW}$

$\mathrm{C}=\mathrm{P} \times \Delta \mathrm{t}$

$\mathrm{C}=9,47 \times 6,53 \times 30$

$\mathrm{C}=1,855 \mathrm{KW}$

$\mathrm{C}_{\mathrm{N}}=1,855 \times 0,38$

$\mathrm{C}_{\mathrm{N}}=\mathrm{R} \$ 705$

Para o regime reduzido tem-se uma redução nos valores da tensão e da corrente em $48 \%$ e $15 \%$ respectivamente. Portanto, tem-se o consumo em RR demonstrado como:

$\mathrm{C}_{\mathrm{R}}=$ Consumo em regime reduzido;

$\mathrm{U}=380 \times 0.52=197,6 \mathrm{~V}$

$\mathrm{I}=29 \times 0,85=24,65 \mathrm{~A}$

$\mathrm{P}=\mathrm{U} \times \mathrm{I} \times \mathrm{FP}$

$P=197,6 \times 24,65 \times 0,86$

$\mathrm{P}=4,18 \mathrm{KW}$

$\mathrm{C}=\mathrm{P} \times \Delta \mathrm{t}$

$\mathrm{E}=4,18 \times 5,92 \times 30$

$\mathrm{C}=742,3 \mathrm{KW}$

$\mathrm{C}_{\mathrm{R}}=742,3 \times 0,38$

$\mathrm{C}_{\mathrm{R}}=\mathrm{R} \$ 282$

Como em regime parado a tensão e a corrente são praticamente nulas concluise que o consumo em regime parado $(\mathrm{CP})$ é nulo.

A economia final $\left(E_{f}\right)$ mensal é dada pela subtração entre o valor gasto antes da implantação do sistema e a soma dos valores gastos $\left(\mathrm{C}_{\mathrm{N}}+\mathrm{C}_{\mathrm{R}}\right)$ com a utilização do projeto SCER.

$\mathrm{C}_{\mathrm{T}}=$ Consumo Total;

$\mathrm{C}_{\mathrm{T}}=\mathrm{C}_{\mathrm{N}}+\mathrm{C}_{\mathrm{R}}$

$\mathrm{C}_{\mathrm{T}}=705+282$

$\mathrm{C}_{\mathrm{T}}=987$ reais

$\mathrm{E}_{\mathrm{f}}=\mathrm{C}_{\mathrm{S}-} \mathrm{C}_{\mathrm{T}}$

$\mathrm{E}_{\mathrm{f}}=1511-987$

$\mathrm{E}_{\mathrm{f}}=524$ reais $=34,6 \%$ 
O resumo dos valores de estimativa de economia com a implantação do SCER está apresentado na Tabela 1.

Tabela 1 - Estimativa de Economia

\begin{tabular}{|l|c|c|}
\hline \multicolumn{2}{|l|}{ Dados de consumo e Economia } \\
\hline Características da Escada & Tipos de consumo & Valor em Reais \\
\hline Motor de 15Hp & CS = Consumo normal (sem o sistema) & R\$ 1.511 \\
\hline Tensão 380V & TN= Tempo em regime normal & CN\$ 705 \\
\hline Corrente 29 A & TR= Tempo em regime reduzido & CR= R\$ 282 \\
\hline Fator de Potência 0,86 & TP= Tempo parada & CP= R\$ 0 \\
\hline & Consumo Total $(C T) \rightarrow C T=C N+C R$ & CT $=$ R\$ 987 \\
\hline & Economia final $($ Ef $) \rightarrow$ Ef $=$ CS- CT & Ef $=$ R\$ 524 $=34,6 \%$ \\
\cline { 2 - 3 } &
\end{tabular}

Com a análise estatística pode-se concluir que existem diferentes fluxos de usuários ao longo do dia, o que torna o sistema de difícil modelagem, o que dificulta encontrar os tempos ótimos de configuração do sistema. Apesar desta característica a análise também demonstrou que o método utilizado foi satisfatório. Como a escada estudada foi a de maior circulação do estabelecimento pode-se concluir que a eficiência do sistema é ainda maior em outras escadas, o que torna o SCER ainda mais atrativo. $\mathrm{O}$ investimento para aquisição do sistema pode ser considerado de baixo custo, sendo o retorno obtido em um curto prazo, estimado em doze meses. Além da economia, pode-se dizer que o sistema é ecologicamente correto. Com a implantação do SCER os gastos com a manutenção dos painéis são reduzidos, uma vez que o painel principal será substituído por componentes mais robustos. Outra melhoria a ser considerada é que todos os dias os funcionários precisam ligar e desligar manualmente as escadas rolantes manualmente, fato este que seria dispensado com a aplicação do sistema.

Para futuras melhorias, pretende-se acoplar ao circuito eletrônico um módulo RTC (Real Timer Clock) a fim de que se tenha uma configuração mais específica para cada dia da semana, o que aumentaria ainda mais a sua eficácia. Este componente trabalharia em conjunto com o microcontrolador, armazenado os tempos de parada e redução de velocidade em seu calendário para cada dia da semana.

\section{CONCLUSÕES}

Pelo estudo realizado verificou-se que a implantação do SCER é viável e de grande valia visto que pode ser implantado em qualquer escada, independente do modelo, marca ou tipo, além de enquadrar-se no contexto de adequação do consumo 
de energia das organizações e redução de impactos ambientais decorrentes da sua geração.

\section{REFERÊNCIAS}

AGÊNCIA NACIONAL DE ENERGIA ELÉTRICA. Manual para elaboração do programa anual de combate ao desperdício de energia elétrica. Disponível em: $<$ http://www.aneel.gov.br/area.cfm?idArea=27>. Acesso em Julho 2007.

BOYLESTAD, R. L.; GUIMARÃES, A. G.; OLIVEIRA, L. A. Dispositivos eletrônicos e teoria de circuitos. 6. Ed. Rio de Janeiro: Livros Técnicos e Científicos, 1999. $649 \mathrm{p}$.

FRANCHI, C. M. Acionamentos Elétricos. 1. ed. São Paulo: Érica, 2007. 250p.

PRADO, D. Teoria das Filas e da Simulação - Série Pesquisa Operacional. 3. ed. Nova Lima: Editora Indg Tecnologia e Serviços LTDA, 2006. 127 p.

SIMÃO, E. B.; ALMEIDA NETO, M. F. Rendimento do conjunto Inversor / Motor de Indução sob diferentes condições de Carga. 2002. Disponível em: $<$ http://www.eee.ufg.br/cepf/pff/2002/ee_14.pdf > . Acesso em: 10 ago. 2007.

ZANCO, W. S. Z. Microcontroladores PIC16F628A/648: Uma abordagem prática e objetiva. 1. Ed. Érica, 2005. 368p.

Manuscrito recebido em:15/02/2009 Revisado e Aceito em:05/08/2009 